\title{
Air sample system optimization for the raw materials industry objects monitoring
}

\author{
M.V. Volkodaeva \\ Professor, Saint-Petersburg Mining University, Saint-Petersburg, Russia \\ Ya.A. Volodina \\ Postgraduate student, Saint-Petersburg Mining University, Saint-Petersburg, Russia \\ V.A. Kuznetsov \\ Postgraduate student, Saint-Petersburg Mining University, Saint-Petersburg, Russia
}

\begin{abstract}
The raw material industry has a negative impact on the environment. In particular, it is an air pollution source. In order to improve the air sampling procedure, it is suggested to use lavsan sample bags. A distinctive feature of these devices is the ease of their use, regeneration and transportation, the possibility of their multiple application and averaging air samples for any period of time. Due to structural features and technical characteristics of bags, the procedure of their delivery to laboratories from remote observing sites is simplified, as well as air samples analysis. To establish the feasibility of using proposed sample bags and to assess the impact of the phosphorite mining enterprise the research was conducted, results of which allow us to conclude that it is advisable to use these devices for sample collection and transportation.
\end{abstract}

\section{INTRODUCTION}

Mineral resources are a complex of raw material stocks in the bowels that can ensure the stable development of the country's economy for a significant future (Boyko et al. 2019, Kozlov et al. 2018). Exploitation of natural resources by a mineral resource sector is associated with the extraction, removal of overburden, ore, coal, oil, gas, groundwater, etc. from geosystems. Attendant problems of the use of natural resources are man-made impacts on environmental compartments that cause their changes (Mammadov et al. 2019, Nevskaya et al. 2019). In this regard, the mineral resource complex has a negative impact on the environment (Krutskikh 2019, Pashkevich et al. 2019), in particular, as a source of emission of a wide range of pollutants into the atmospheric air. The main pollutants in the air basin are particulate pollutants, sulfur dioxide, carbon monoxide, nitrogen oxides and volatile organic compounds (State report 2018).

In the total volume of pollutant emissions into the atmosphere from stationary sources, which amounted to $17,476.2$ thousand tons in 2017 , the largest contribution was made by sources (by type of economic activity): "Manufacturing" - 5802.2 thousand tons, or $33.2 \%$ of all emissions from stationary sources; "Mining" - 4,918.9 thousand tons, or 28.1\%; "Provision of electric energy, gas and steam; air conditioning"- 3,542.6 thousand tons, or 20.3\% (State report 2018).

Therefore, in order to carry out effective environmental protection activities, it is necessary to be provided with sufficient information about the state of the environment, take into account spatial and temporal factors, the degree of anthropogenic impact, anticipate the possible consequences of human intervention in the course of natural processes. The tool for obtaining such information is environmental monitoring (State report 2018). 


\section{ENVIRONMENTAL MONITORING SAMPLING}

Monitoring conducted by enterprises to assess the impact of their activities on the natural environment (and, first of all, on the most sensitive component of ecosystems - atmospheric air) can be both instrumental and calculated. Calculated monitoring allows you to determine the level of pollution based on the data on emissions of pollutants. Thanks to this approach, it is possible to estimate the level of pollution not only at its source, but also at any distance from the industrial facility. Instrumental monitoring involves sampling on the ground (usually in the area of greatest pollution) and subsequent laboratory analysis, allowing you to obtain data on the level of air pollution in the zone of a particular enterprise influence. However, it should be noted that the largest measurement errors occur at the stage of sampling of atmospheric air (Watson et al. 2011). Also carrying out full-fledged instrumental monitoring is associated with a number of difficulties, for example, the inability to quickly obtain data from remote areas, the operation of obsolete equipment, as well as sampling methods and devices (Yakunina \& Popov 2009).

Basically, sampling methods are divided into 2 groups: aspiration (air suction through absorption solutions, filters, sorption tubes or grain sorbents placed in the absorber) and sampling in vessels (bottles, gas pipettes and other containers) that are delivered for the further analysis to the laboratory (Woolfenden 2010).

Air sampling is most often carried out by the aspiration method in order to determine the concentration of chemical compounds. The method is based on aspiration of a known volume of air through an absorption material capable of retaining substances to be determined. This method is used when it is necessary to concentrate the microimpurities in the absorber and to increase the sensitivity of the analysis method.

As an absorption solution, distilled water or special liquids that dissolve toxic substances or interact with them are used. Absorbers with solid sorbents are also used: silica gel, activated carbon, and others. They have a different design: in them, solid sorbents can be stationary or set in motion by an air current, forming a "fluidized bed", which contributes to a greater contact of the sorbents with air and improves the absorption of the substance of interest. Sorption tubes are also used to take and analyze air samples. They are filled with glass powder, impregnated with special solutions for trapping toxic substances. This type of sampling suction systems is most common for environmental monitoring tasks.

For quick sampling with the availability of sensitive research methods, sampling into various vessels and containers is used. The type of gas sampling vessel is determined by the nature of the gas being analyzed and the volume of the sample. It is noteworthy that the volume of the gas sample should be sufficient for analysis by the selected method and for repeated determinations.

Sample storage conditions are an important factor in the analysis of environmental component. For example, air samples taken on active solid sorbents can be stored for a limited time, in some cases cooling is necessary. Air samples taken in vessels are not recommended to be stored for a long time, since reactions with oxygen, water vapor, adsorption of the vessel walls and so on are possible. One of the main problems in determining the concentrations of volatile substances is associated with this.

Typically, gas samples are taken into the device in which the analysis is performed. If the analysis cannot be performed at the sampling site, air samples are delivered to the laboratory. Gas diffusion through some connecting tubes may cause a change in the composition of the gas sample. The composition of the average sample may vary in the sampling device itself. Another important factor is the temperature - a significant temperature difference at the time of selection and during storage can significantly affect the final result of the analysis. Therefore, when optimizing sampling methods, various aspects should be considered that affect the concentration of pollutants in the sample.

Currently, various devices are used in the Russian Federation for air sampling: direct sorption and indicator tubes, various types of absorption vessels (for example, Zaitsev, Richter vessels with a porous plate), cooled traps, syringes, gas pipettes and others. Most of these devices have several disadvantages. For example, when using sorption tubes, the concentration of substances in the sample decreases as a result of adsorption by the tube material, and during subsequent analysis of the sample, false peaks may appear on the gas chromatogram (as a result of desorption of 
previously adsorbed substances). The use of syringes and gas pipettes does not comply with the regulatory and technical documentation for sampling (due to the time of sampling), as well as these devices do not allow storing air samples. Moreover, many sampling devices require considerable time for their regeneration, as well as the preliminary preparation of special sorbents and absorption solutions. These shortcomings indicate the need for modernization of the monitoring network, in particular, the introduction of new methods and devices for air sampling for the subsequent determination of the pollutant content in the air.

It should be noted that in recent years, sample bags have increasingly been used for sampling around the world (Watson et al. 2011). They are devices for sampling air, which are used both for basic gases and for specific substances. These bags can be made of various materials (teflon, lavsan, fluoroplastic, polyvinylidene fluoride, fluoroethylene propylene, aluminum film, etc.). The leaders in the production of sampling bags are considered to be Restek (USA), SKC (USA), ColeParmer (USA), Merck (Germany), GL Sciences (The Netherlands).

In support of the feasibility and advisability of using these sampling devices, many studies have been carried out around the world. For instance, some scientists consider the problem of storage stability (Akdeniz et al. 2011, Laor et al. 2010); in other works the question regarding the sample loss is raised (Sironi et al. 2014, Kim \& Kim 2012); there are also some works on the different types of bags (Kim et al. 2012, Laor et al. 2010) and materials (Salo \& Makinen 2019) comparison. Of particular interest is the study of comparing commercial and homemade bags (Parker et al. 2010, Abruzzi et al. 2019). All in all, the results allowed concluding that air sample bags can be an alternative to the widely used sampling devices and are suitable for many areas, including industrial hygiene, landfill (biogas), ambient air, indoor air and stationary source testing. The disadvantage of these devices is their high cost, often the inability to reuse, as well as the inability to store some volatile organic compounds.

For the needs of industrial environmental control and atmospheric air monitoring in the Russian Federation, which includes the regular determination of the pollutant concentrations over immense territories and in remote areas, as well as for studying the content of specific compounds in samples, there is no universal sampling device. This is due to the need to fulfill a number of conditions: ease of sampling, ease of the device regeneration, a low background level of substances in the device, economic affordability, the possibility of long-term storage of samples and comparisons with established environmental standards.

In order to improve the atmospheric air sampling procedure, the authors propose the use of lavsan sample bags, which allow subsequent determining of the concentrations of pollutants that are characteristic of mineral resource complex enterprises. Due to the design features and technical characteristics of the bags, the procedure of their delivery to laboratories from remote observation points and analysis of air samples is simplified, and the cost of devices is reduced.

To establish the feasibility and advisability of using the proposed sample bags with the participation of the authors numerous studies were carried out: the concentrations of pollutants at control points without sampling were determined and also in laboratory conditions with preliminary sampling of atmospheric air into the lavsan bags (Volkodaeva \& Volodina 2017); the optimal methods for the sample bags regeneration were identified (Volkodaeva et al. $2019 \mathrm{~b}$ ); the possibility of using these devices at the border of the sanitary protection zone was demonstrated (Volkodaeva et al. 2019a).

\section{SAMPLE BAGS APPLICATION}

Sample bags made of polymer films are intended to be used for the collection and storage of gaseous samples for subsequent analysis on laboratory equipment (chromatographs, stationary gas analyzers, etc.). The bags can be used to control the pollution of the atmosphere, air of the working area, industrial emissions, as well as to control production processes in the chemical, petrochemical, pharmaceutical, food industries, medicine, agriculture, etc.

Sampling in bags is carried out by means of portable sampling pumps or vacuum samplers. The capacity of the packages can vary from 0.5 to 100 liters. The bags are equipped with 
various fittings: stainless steel, polypropylene, teflon, designed both for sampling from the bag with a syringe, and using an external pump. The number of fittings may also vary.

Compared to other sampling devices (gas pipettes, gas syringes, etc.), bags of polymer films have several advantages:

- flexibility: a variety of organic and inorganic compounds can be sampled and stored in bags;

- high stability of sample storage;

- the ability to automatically average samples over a 20-minute (or other) time interval;

- the ability to automatically adapt the sample to the conditions of analysis (temperature and pressure);

- the variety of fitting types and their quantity;

- lightness, strength and durability.

A study of a great variety of sample bags produced by world leaders, as well as Russian manufacturers, showed that there is no appropriate device for the collection, storage and transportation of samples of volatile organic compounds (control of such compounds is mandatory in large cities and near enterprises-sources of these substances) for the purpose of environmental monitoring of atmospheric air. Some samples are disposable and cannot be regenerated, others are not able to hold the specific contaminants necessary for determination, and others are equipped with fittings that are inconvenient to use. Thus, the absence of a suitable sampling device leads to the search for technical solutions to optimize the procedure of sample collection, storage, transportation, as well as the procedure of bags regeneration.

Currently, the monitoring of the content of volatile organic compounds in the air is carried out over vast territories. It implies sampling on site and delivering air samples to specialized laboratories. Due to the remoteness of some observation points, as well as the load of analytical equipment in laboratories, the solution is to increase the storage time of samples in bags.

The authors propose to make some changes to the design of the sample bag, namely, to improve the seams and fittings of the device. The use of reinforced double seams has a positive effect on the duration of storage of samples and less diffusion of pollutants through the seams of the bag - the most fragile part of the sampling device. There is also a need for better cleaning of devices, which can be achieved by identifying the optimal regeneration method, as well as placing an additional fitting on the opposite side of the bag.

\section{SAMPLE BAG DESIGN OPTIMIZATION}

The authors propose to make changes to the design of the sample bags, as well as to use the identified effective ways of regenerating these devices.

The manufacture of sample bags from an ultra-pure lavsan film with a thickness of $50 \mu \mathrm{m}$ can be considered the optimal solution, because with the small weight of the bag the barrier functions necessary for storing the samples are performed. The material prevents the diffusion of gases through the walls of the bag, which is similar to preserving the air sample inside the device. The usage of double seams increases the storage time of volatile organic compounds in atmospheric air samples. Seams are made by the agency of a welding machine.

Moreover, it is also possible to place a fluoroplastic tube with a diameter of $1-2 \mathrm{~mm}$ in the space between two seams (Figure 1). This tube, located around the entire perimeter of the bag, will make it possible to make a kind of frame and at the same time reduce the likelihood of deformation of the device and, as a result, loss of atmospheric air sample.

The use of two polypropylene fittings increases the "flexibility" of the sampling. In other words, it allows taking samples in different conditions, varying the way the bag is attached to the sample feeder and the sampling speed, and also improves the quality of the bag regeneration by the through-bag purging.

Another feature of lavsan sample bags is the possibility of their multiple use. For the purpose of this, devices should be regenerated properly. The authors propose the use of ozone and nitrogen as gases for cleaning bags. The choice of a regeneration method is based on the physicochemical characteristics of substances acting as purifiers (Volkodaeva et al. 2019b). 


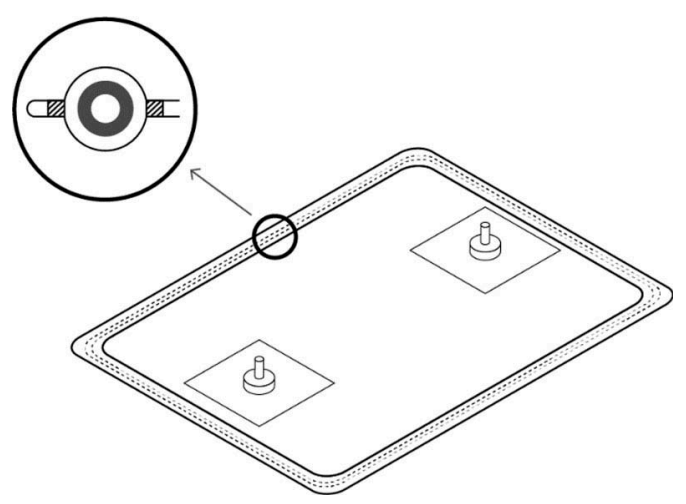

Figure 1. Sample bag with a fluoroplastic tube.

From an environmental point of view, lavsan material can be recycled and disposed of. There are two main ways of processing polyethylene terephthalate: mechanical and physicochemical; biological methods are actively developing (Yoshida et al. 2016). Moreover, polypropylene processing is practiced. It includes the stages of sorting, crushing and temperature exposure, granulation.

Thus, the proposed characteristics of the sample bags make it possible to obtain reliable data from remote areas (relevant for objects of the mineral resource complex), as well as to conduct numerous sampling while monitoring air quality at the border of the sanitary protection zone. It should be noted that there are various ways to recycle and dispose of the material from which the bags are made.

\section{FIELD STUDY}

To assess the impact of the phosphorite mining enterprise, measurements were made on site and 20-minute air samples with an hourly interval during the active phase of the production process were taken. As a part of the study, the concentrations of the following pollutants were determined: benzene, toluene, ethylbenzene, isomers of xylene, styrene, acetone.

In order to obtain more complete and representative environmental information about the state of the air basin, as well as to conduct a comparative experiment, the authors decided to use several different devices for sampling (in addition to direct measurements on site). Such devices were sorption tubes (filled with Carbopack ${ }^{\mathrm{TM}} \mathrm{B}$ ), Tedlar ${ }^{\circledR}$ sample bags (manufactured by SKC) and homemade lavsan sample bags. The measurements on site were carried out by the agency of portable gas chromatograph FGH (manufactured by Research and Production Company Ekan).

Samples were taken in an open field, without obstacles to capture pollutants from the leeward side of the source of pollution at the border of the sanitary protection zone.

After sampling, all samples were delivered to the laboratory within three hours for further analysis (by means of a gas chromatograph FGH).

The averaged results of determining the pollutant concentrations in different ways are presented in Table 1 and Figure 2.

The results of direct measurements made on site should be considered as reference, since such measurements do not have factors affecting the sample such as its storage and transportation. Moreover, background concentrations of sampling devices can also distort the final result.

According to the research, all the devices used provide fairly good stability of the samples during their transportation and preservation of volatile organic compounds. However, there is a decrease in the concentration of pollutants in the samples due to 
Table 1. Averaged results of determining the pollutant concentrations in samples of atmospheric air.

\begin{tabular}{lllll}
\hline Pollutant & $\begin{array}{l}\text { Direct measurements } \\
\text { (on site) }\end{array}$ & $\begin{array}{l}\text { Preliminary } \\
\text { sampling in } \\
\text { sorption tubes }\end{array}$ & $\begin{array}{l}\text { Preliminary } \\
\text { sampling in } \\
\text { Tedlar }^{B} \text { bags }\end{array}$ & $\begin{array}{l}\text { Preliminary } \\
\text { sampling in } \\
\text { lavsan bags }\end{array}$ \\
\hline benzene $(\mathrm{mg} / \mathrm{m} 3)$ & 0.023 & 0.011 & 0.015 & 0.018 \\
toluene $(\mathrm{mg} / \mathrm{m} 3)$ & 0.091 & 0.124 & 0.065 & 0.071 \\
ethylbenzene $(\mathrm{mg} / \mathrm{m} 3)$ & 0.018 & 0.009 & 0.012 & 0.015 \\
m-xylene $(\mathrm{mg} / \mathrm{m} 3)$ & 0.184 & 0.104 & 0.122 & 0.151 \\
p-xylene $(\mathrm{mg} / \mathrm{m} 3)$ & 0.159 & 0.113 & 0.135 & 0.142 \\
o-xylene $(\mathrm{mg} / \mathrm{m} 3)$ & 0.078 & 0.043 & 0.061 & 0.068 \\
styrene $(\mathrm{mg} / \mathrm{m} 3)$ & 0.002 & $<0.001$ & 0.001 & 0.002 \\
acetone $(\mathrm{mg} / \mathrm{m} 3)$ & 0.016 & 0.007 & 0.004 & 0.011 \\
\hline
\end{tabular}

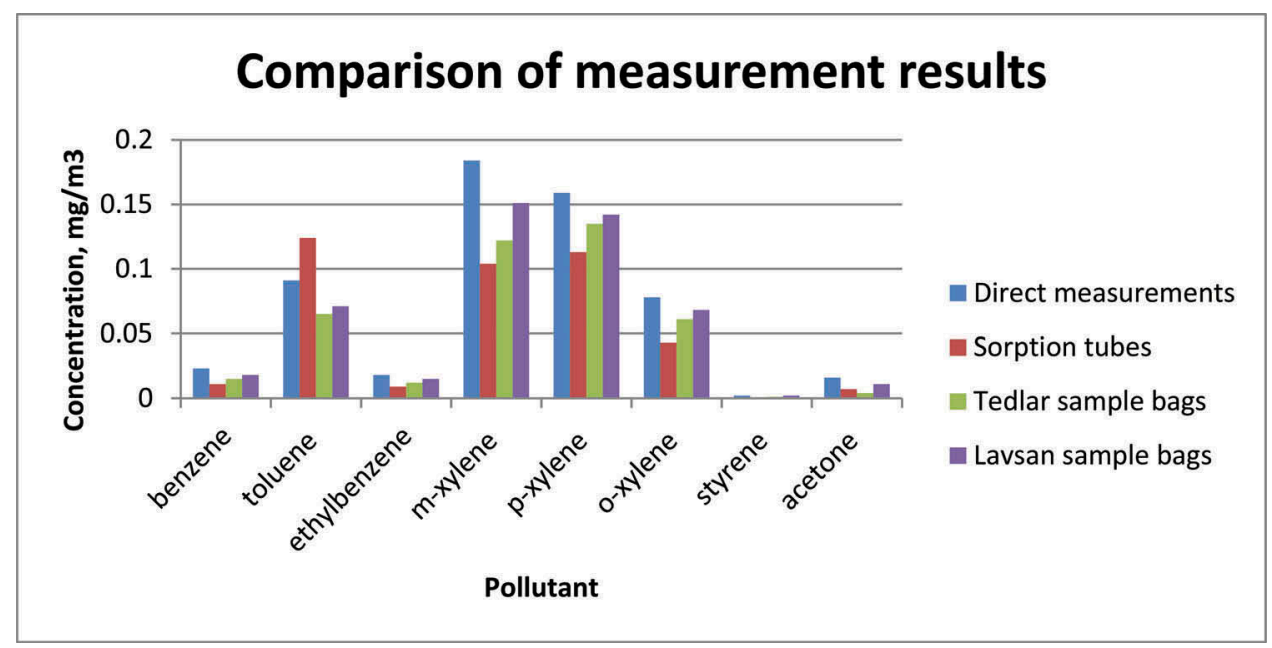

Figure 2. Comparison of results of a quantitative measurement of pollutants.

volatilization, adsorption (in the case of tubes) or deposition (on the inner walls of the bags). An exception is the result of the determination of toluene, collected using sorption tubes, which may be caused by the desorption of previously adsorbed substances. The correlation coefficient between the results of direct measurements and measurements using sorption tubes amounted to 0.86 , Tedlar ${ }^{\circledR}$ bags -0.98 , lavsan bags -0.99 . The discrepancies between the values obtained during measurements on site and during the analysis of samples taken in bags under laboratory conditions vary within a few tens of percent, which is acceptable when conducting such studies.

All in all, the results obtained during the research allow us to conclude that it is possible and advisable to use devices for sampling and transportation of samples, in particular, lavsan sample bags, in order to control the quality of atmospheric air. Despite a slight decrease in pollutant concentrations in lavsan bags, the use of these devices for the purposes of environmental monitoring is a good alternative to outdated methods.

It should also be noted that the results of this study, similar to the results reflected in the Abruzzi's research (Abruzzi et al. 2019), show that homemade containers are a good alternative to commercial ones for storing air samples and further analysis by chromatographic method. The Abruzzi's work also says about the possibility of stable storage of samples in homemade borosilicate glass containers for up to 96 hours, while in our lavsan bags the time of stable storage is about 72 hours. However, lavsan bags are also suitable for volatile organic compounds sampling and it is easier to transport and store them due to their light weight and shape. 
Moreover, Kim's research (Kim et al. 2012) on the storage of volatile organic compounds in polyester aluminum (PEA) and polyvinyl fluoride (PVF) sample bags showed more stable storage of substances in PEA containers than in PVF ones. Homemade lavsan sample bags showed similar results in our research.

\section{CONCLUSION}

The raw material industry has a significant negative impact on the environment and atmospheric air, in particular. In this regard, it is mandatory to conduct continuous air quality monitoring both at the facilities themselves and at the border of the sanitary protection zone, which implies sampling. Since widely used methods and devices have certain drawbacks, the authors propose the use of lavsan sample bags. When using these bags, it becomes possible to average the air samples over different time intervals, to deliver the samples from distant observation points to the laboratory for subsequent analysis. Bags can be used multiply, which is also a cost-effective solution for monitoring systems of different levels. In support of the feasibility and advisability of using these sampling devices, many studies have been carried out around the world.

In a similar vein authors conducted an experiment in order to compare different sampling devices - sorption tubes, manufactured and homemade sample bags. According to the research, all the devices used provide fairly good stability of the samples during their transportation and volatile organic compounds preservation. The discrepancies between the values obtained during measurements on site and during the analysis of samples taken in bags vary within a few tens of percent, which is acceptable when conducting such studies. Homemade lavsan sample bags also showed good results and, consequently, could be used for environmental monitoring purposes despite a slight decrease in pollutant concentrations in them.

Thus, the optimization of the sampling system through the introduction of specialized bags will improve the quality and speed of obtaining reliable information necessary for decision-making on environmental protection, as well as assessing effectiveness of nature protection measures.

\section{REFERENCES}

Abruzzi, R.C., Bonetti, B., Marçal, J.R.P., Berenice, A.D. \& Bitencourt, A.K. 2019. Artifacts in the analysis and assessment of low-cost containers for sampling and storing greenhouse gases. Quimica Nova 42(1): 84-94.

Akdeniz, N., Janni, K.A., Jacobson, L.D. \& Hetchler, B.P. 2011. Comparison of gas sampling bags to temporarily store hydrogen sulfide, ammonia, and greenhouse gases. Transactions of the ASABE 54: 653-661.

Boyko, N.A., Chvileva, T.A. \& Romasheva, N.V. 2019. The impact of coal companies on the socio-economic development of coal mining regions and its assessment. Ugol 11(1124): 48-53.

Kim, Y.-H. \& Kim, K.-H. 2012. Experimental approach to assess sorptive loss properties of volatile organic compounds in the sampling bag system. Journal of Separation Science 35(21): 2914-2921.

Kim, Y.-H., Kim, K.-H., Jo, S.-H., Jeon, E.-C., Sohn, J. R. \& Parker, D. B. 2012. Comparison of storage stability of odorous VOCs in polyester aluminum and polyvinyl fluoride Tedlar ${ }^{\circledR}$ bags. Analytica Chimica Acta 712: 162-167.

Kozlov, A.V., Teslya, A.B. \& Chernogorsky, S.A. 2018. Game Theory Model of State Investment into Territories of Advanced Development in the Regions of Mineral Resources Specialization. Journal of Mining Institute 234: 673-682.

Krutskikh, N.V. 2019. Assessment of nature transformation in the mining influence zone by earth remote sensing data. Gornyi Zhurnal 3: 88-93.

Laor, Y., Ozer, Y., Ravid, U., Hanan, A. \& Orenstein, P. 2010. Methodological aspects of sample collection for dynamic olfactometry. Chemical Engineering Transactions 23: 55-60.

Mammadov, V.A., Salamov, A.M. \& Khalilova, H.Kh. 2019. Study of the anthropogenic impact on the change of geoecological conditions of the Khojahasan lake, Azerbaijan. Journal of Mining Institute 239: 603-610. 
Nevskaya, M.A., Seleznev, S.G., Masloboev, V.A., Klyuchnikova, E.M. \& Makarov, D.V. 2019. Environmental and business challenges presented by mining processing waste in the Russian Federation. Minerals 9(7): 445.

Parker, D.B., Perschbacher-Buser, Z.L., Cole, N.A. \& Koziel, J.A. 2010. Recovery of agricultural odors and odorous compounds from polyvinyl fluoride film bags. Sensors 10(9): 8536-8552.

Pashkevich, M.A., Matveeva, V.A. \& Danilov, A.S. 2019. Migration of pollutants from the mining waste disposal territories on the Kola Peninsula. Gornyi Zhurnal 1: 17-21.

Salo, H. \& Makinen, J. 2019. Comparison of traditional moss bags and synthetic fabric bags in magnetic monitoring of urban air pollution. Ecological indicators 104: 559-566.

Sironi, S., Eusebio, L., Capelli, L., Boiardi, E., Del Rosso, R. \& Guillot, J.M. 2014. Ammonia diffusion phenomena through Nalophan bags used for olfactometric analyses. Journal of Environmental Protection 5: 949-961.

State report. 2018. On the State and Environmental Protection of the Russian Federation in 2017. Moscow: Ministry of Natural Resources and Environment of the Russian Federation, Research and Production Enterprise Kadastr.

Volkodaeva, M.V. \& Volodina, Y.A. 2017. Comparing the results of measurements of concentrations of volatile organic compounds using different sampling methods. Ecological systems and devices 7: 3-6.

Volkodaeva, M.V., Volodina, Y.A. \& Kuznetsov, V.A. 2019a. Development of industrial environmental control methods. IOP Conf. Series: Earth and Environmental Science 378(2019) 012108.

Volkodaeva, M.V., Volodina, Y.A. \& Kuznetsov, V.A. 2019b. On the selection of the lavsan sample bags regeneration for their repeated use. Danish Scientific Journal 21(1): 65-68.

Watson, N., Davies, S., Wevill, D. 2011. Air Monitoring: New Advances in Sampling and Detection. Scientific World Journal 11: 2582-2598.

Woolfenden, E. 2010. Sorbent-based sampling methods for volatile and semi-volatile organic compounds in air - part 1: sorbent-based air monitoring options. Journal of Chromatography A. 1217(16): $2674-2684$

Yakunina, I.V. \& Popov, N.S. 2009. Methods and instruments for environmental control. Environmental Monitoring: A Training Manual. Tambov: Publishing house of Tambov State technical university.

Yoshida, S., Higara, K., Takehana, T., Taniguchi, I., Yamaji, H., Maeda, Y., Toyohara, K., Miyamoto, K., Kimura, Y. \& Oda, K. 2016. A bacterium that degrades and assimilates poly(ethylene terephthalate). Science 351: 1196-1199. 